\title{
Educación, libertad y justicia en Fichte
}

Héctor Oscar Arrese Igor

\section{OpenEdition}

\section{Journals}

Edición electrónica

URL: http://journals.openedition.org/ref/762

ISSN: 2258-014X

Editor

EuroPhilosophie Editions

Referencia electrónica

Héctor Oscar Arrese Igor, «Educación, libertad y justicia en Fichte », Revista de Estud(i)os sobre Fichte [En línea], 15 | 2017, Publicado el 01 diciembre 2017, consultado el 13 noviembre 2018. URL : http:// journals.openedition.org/ref/762

Este documento fue generado automáticamente el 13 noviembre 2018.

(c) EuroPhilosophie 


\title{
Educación, libertad y justicia en Fichte
}

\author{
Héctor Oscar Arrese Igor
}

1 Isaiah Berlin reactualizó un antiguo debate acerca de la forma más deseable y justa de la libertad, distinguiendo entre la libertad positiva y la negativa. La libertad negativa consiste en la ausencia de coerción externa sobre el agente, de modo tal de que pueda llevar a cabo los fines que desee en ese espacio privado. ${ }^{1}$ Por otro lado, Berlin define a la libertad positiva como la persecución de algún ideal de vida buena, de la cual quedarían excluidos quienes no compartan esta concepción. ${ }^{2}$ En este sentido, Berlin se ubica en la tradición liberal, en la medida en que rechaza aquellas concepciones de la libertad positiva que implican la imposición de una concepción moral dominante sobre las minorías. Se trata de la crítica de Benjamin Constant a la teoría de Rousseau, entendida como un planteo autoritario que busca avasallar el plano de la privacidad. ${ }^{3} \mathrm{O}$ de las objeciones de John Stuart Mill a la "dictadura de la mayoría", con la consecuente necesidad de proteger la particularidad de cada individuo. ${ }^{4}$

2 Tomando esta clasificación como marco de referencia, Berlin objetó la concepción de la libertad desarrollada por Fichte en su Fundamento del derecho natural. En su texto Freedom and its Betrayals, Berlin argumenta que en la teoría de Fichte no hay lugar para la libertad negativa, dado que el individuo queda subsumido en la totalidad de la comunidad política. ${ }^{5}$ Berlin apoya su interpretación en aquel pasaje en el que Fichte identifica a la comunidad política como una totalitas y no como un mero compositum de individuos. ${ }^{6}$

3 Frente a la interpretación de Berlin, intentaré mostrar en este trabajo que la concepción fichteana de la libertad no puede ser interpretada en términos de las categorías de las libertades negativa y positiva. La razón de esta afirmación reside en que la idea de la libertad en Fichte está íntimamente ligada a la propiedad, dado que se funda en el derecho a la existencia de todos los ciudadanos. Esta concepción implica una idea particular de la justicia distributiva, que no gira en torno de la distribución de bienes, sino de oportunidades para poder vivir del propio trabajo.

4 Ahora bien, también apunto en este trabajo a explorar otra dimensión importante de la idea de la libertad en Fichte, que tiene que ver con el enfoque basado en el desarrollo de 
capacidades. Para ello, en primer lugar reconstruiré la demostración del reconocimiento del otro a partir de la figura humana del Yo en cuanto cuerpo en reposo. Finalmente, derivaré las consecuencias que tiene esta concepción de la libertad como desarrollo de las capacidades para la política educativa diseñada por Fichte.

\section{Libertad y propiedad}

5 La idea de la libertad política en el Fundamento del derecho natural (de ahora en más GNR) de Fichte es un concepto complejo, porque implica una serie de dimensiones diversas. Por un lado, la libertad está unida inextricablemente a la propiedad pero, por el otro, depende de una concepción de la educación de las capacidades para realizar una serie de fines, en el sentido de Bildung. En última instancia, la idea fichteana de la libertad tiene como condicionante último a la educación en capacidades determinadas, dado que la propiedad depende de que los ciudadanos estén en condiciones de vivir de su trabajo.

Dada la multidimensionalidad de la idea fichteana de la libertad, considero que es necesario entrar con cierto detalle en su estructura. En primer lugar, creo que podemos encontrar un momento distributivo en el concepto de libertad, dado que claramente descansa en un determinado sistema de derechos de propiedad. Veamos cómo Fichte llega a esta noción. Cuando Fichte establece el principio del derecho, apunta en última instancia a delimitar esferas iguales de libertad de acción para los sujetos. Es decir que cada uno es libre en la medida en que el otro tiene el mismo campo de acción para tomar decisiones por sí mismo. ${ }^{8}$

7 El fundamento de esta simetría reside en que cada uno de los sujetos puede ser reconocido por el otro como un ser racional en la medida en que el otro le deja un campo libre de acción. La razón de esto es que el otro no puede reconocer al Yo como un ser racional si al mismo tiempo lo coacciona para que actúe de una determinada manera. Se trata de un argumento de tipo pragmático, dado que la estructura de una acción coercitiva presupone que el otro no es un ser libre y que, por lo tanto, puede ser tratado como un objeto. $Y$, por otro lado, Fichte entiende a la relación de reconocimiento como un tipo de acción en la que el otro le transmite al Yo su concepto de un ser racional y libre. ${ }^{9}$

8 Asimismo, cuando el otro exhorta al Yo a la acción libre, lo está reconociendo si bien de modo problemático, dado que todavía falta la respuesta del Yo. El Yo hace posible que el otro lo confirme categóricamente cuando se autodetermina a la acción, respondiendo a la exhortación del otro. De este modo, es necesario que el reconocimiento consista en una influencia recíproca entre los sujetos involucrados en la relación en cuestión. ${ }^{10}$ De este modo, el principio del derecho viene a garantizar esta relación, que por otro lado es constitutiva de la subjetividad.

Ahora bien, resta saber en qué consiste esa esfera de acción que los sujetos deben garantizarse mutuamente. Para determinarla, es necesario en primer lugar aclarar que las esferas de acción en realidad consisten en las condiciones externas de la autodeterminación a la acción. Fichte determina estas condiciones a partir del análisis de la estructura misma de la acción libre. En cuanto acción ella debe estar estructurada teleológicamente. Esto significa que la acción se define por el fin que el agente desea realizar.

10 Con esta idea en mente, Fichte analiza las condiciones que permiten al agente proponerse el fin de realizar la acción. La más importante de ellas es la garantía que el agente debe 
tener de que al menos seguirá existiendo al momento de realizar la acción. Por lo tanto, el agente persigue con cada acción el objetivo fundamental de la auto-conservación en la existencia. ${ }^{11}$ Ahora bien, Fichte debe resolver el problema del status de esta búsqueda.

11 En primer lugar, Fichte rechaza la fundamentación de esta tendencia a la autoconservación en un deseo a ser realizado en el futuro. Si la acción tuviera como un elemento constitutivo central este deseo futuro, entonces no podría explicarse esta búsqueda de la auto-conservación. El problema de esta estrategia radica en que no puede pensarse la acción como estructurada en torno de un determinado deseo del futuro, porque esta acción misma también sería producto de un deseo del futuro, por lo que la explicación caería en un regreso al infinito. Por lo tanto, Fichte emprende una estrategia fundada en la identificación de algún mecanismo constitutivo del cuerpo humano que provoque el impulso a garantizarse la auto-conservación en la existencia.

Fichte concibe al cuerpo como una totalidad organizada teleológicamente, cuyo móvil ( Triebfeder) fundamental consiste en la supervivencia del organismo. Dado que el cuerpo es la condición de posibilidad de toda acción, entonces el móvil fundamental de toda acción es la auto-conservación del cuerpo. Por lo tanto, el derecho debe garantizar la hidratación y nutrición de todos los ciudadanos, a fin de preservar su libertad de acción. De lo argumentado hasta aquí Fichte concluye que

[e]l fin supremo y universal de toda actividad libre es, por lo tanto, poder vivir. Todo el mundo tiene este fin, y está garantizado en la misma medida en que está garantizada la libertad en general. Sin su obtención no serían posibles en absoluto ni la libertad ni la permanencia de la persona. ${ }^{12}$

13 Todo el sistema legal fichteano parece pues girar en torno de la garantía del derecho a la existencia. Efectivamente, considero que el contrato de propiedad, que es el primer momento del contrato de ciudadanía, puede ser interpretado en estos términos. El contrato de propiedad es aquel momento en el que los ciudadanos se comprometen a no interferir en la esfera de acciones de los demás. ${ }^{13}$ Dicho de otro modo, cada uno se abstiene de impedir al otro la realización de los fines que pueda proponerse llevar a cabo en el mundo sensible. A partir de lo argumentado más arriba, esto implica que cada uno debe evitar poner obstáculos para que los demás no satisfagan su derecho a la existencia.

Esta exigencia se traduce en el siguiente derecho natural: "todo el mundo debe poder vivir de su trabajo". ${ }^{14}$ De este modo, la unidad de distribución de la propiedad es la profesión o el oficio que cada ciudadano desempeñe. Este es el contenido concreto de su esfera de acción. Toda vez que la acción tiene como último horizonte la satisfacción de las necesidades vitales del agente, cada ciudadano debe poder ganarse el pan con su trabajo. Como señala acertadamente Zwi Batscha, el concepto de ciudadanía en Fichte no es algo abstracto, es decir que no se trata de una serie de características compartidas entre todos los ciudadanos tales como, por ejemplo, ser nativo del país que habita o haber cumplido con los requisitos legales para gozar de una serie de derechos universales. Por el contrario, siguiendo a Batscha, la ciudadanía está constituida intrínsecamente por aquella tarea que cada uno desempeña en el marco de la división social del trabajo. Por lo tanto, el status social del individuo es aquello que lo define como ciudadano. ${ }^{15}$

En este marco Fichte pone un ejemplo ilustrativo. Imaginemos, argumenta Fichte, un pueblo de ciudadanos que anden desnudos. En este pueblo no tendría sentido permitir a un ciudadano que ejerza el oficio de sastre, porque nadie podría vivir allí de ese trabajo. ${ }^{16}$ Por el contrario, si el Estado debe hacer posible que el sastre viva de su trabajo, deberá exigir que los ciudadanos compren sus trajes. Por lo tanto, si el Estado define la 
ciudadanía de sus miembros en función de su oficio, debe garantizar a los mismos las condiciones para llevarlo a cabo. En este sentido, si un ciudadano no llega a poder vivir de su trabajo, entonces el Estado debe apoyarlo para conseguir otra tarea de la que pueda vivir. ${ }^{17}$ El respeto por la propiedad del ciudadano implica la reproducción de las condiciones que hacen posible dicha propiedad.

En la teoría fichteana del Estado cada ciudadano está inserto en una red de relaciones con los otros de modo tal que su liberad externa está condicionada por la de todos los demás. Por esa razón el Estado tiene la atribución de controlar el modo en que se producen los bienes y el modo en que estos son distribuidos entre los ciudadanos. ${ }^{18}$ Esta atribución no puede ser delegada al libre mercado dado que, desde el punto de vista de Fichte, se trata de un sistema azaroso de producción e intercambio de bienes que no está en condiciones de garantizar los derechos de nadie.

En consecuencia cada ciudadano tiene derecho a coaccionar a todos los demás a través de las instituciones del Estado para que cumplan con el compromiso de garantizarle el ejercicio de aquella profesión que ha declarado ejercer. En caso contrario, el contrato de ciudadanía carece de validez. Esto acarrea consecuencias aún más importantes, dado que, en el caso de que un ciudadano no vea garantizado su derecho a la existencia, no tendrá la obligación de respetar la propiedad de los demás. Esto implica a su vez que todas las propiedades de los demás pertenecen al ciudadano que está pasando necesidad, dado que deben ser redistribuidas por el Estado. Dicho de otro modo: si un solo ciudadano ve en peligro su subsistencia, esto conlleva una hipoteca sobre los bienes de los demás. ${ }^{19}$

Como señala Allen Wood, la propuesta de Fichte es novedosa para su época porque fundamenta una concepción de la justicia distributiva pero en términos liberales, en el sentido que adquirirá esta palabra en el siglo XIX. Es decir que Fichte entiende que el Estado debe redistribuir los bienes según el criterio de las necesidades vitales, pero teniendo en mente el derecho inalienable de los ciudadanos a llevar su vida privada según la concepción de la vida buena que quieran sostener en cada caso. Por lo tanto, como señala acertadamente Wood, en la teoría fichteana el Estado debe garantizar que los ciudadanos no lleguen a padecer un estado tal de pobreza que los lleve a depender del arbitrio de los demás, para poder sobrevivir. De este modo, Fichte argumenta que quienes estén en una situación de vulnerabilidad estarán a merced de las interferencias arbitrarias de los demás. Finalmente, según la interpretación de Wood, Fichte intenta también reducir la posibilidad de que algunos ciudadanos acumulen tal cantidad de poder o de riqueza que los habilite para reducir la libertad de los demás, estableciendo relaciones de dominación. ${ }^{20}$

19 Por otro lado, la idea de un derecho a la existencia conlleva una reinterpretación del contrato de protección, que es el segundo momento del contrato de ciudadanía. Según las cláusulas del contrato de protección, cada ciudadano debe cooperar activamente para proteger la propiedad de los demás. ${ }^{21}$ En este sentido, si la propiedad fundamental de los ciudadanos es el derecho a vivir de su trabajo, entonces todos deberán ser coaccionados para que entreguen sus propiedades en el caso eventual de que los demás las necesiten para subsistir.

Ahora bien, la idea de un derecho a la existencia tiene consecuencias de peso incluso para cuestiones que podrían considerarse como propias del ámbito privado. El derecho a la autoconservación en la existencia no implica solamente que el Estado redistribuya la propiedad de modo tal que los ciudadanos reciban lo necesario para poder vivir de su trabajo. También este derecho exige que los ciudadanos puedan administrar dichos bienes 
de modo tal de que puedan lograr con ellos la autoconservación que se persigue. De este modo, el Estado tiene la obligación de controlar si los ciudadanos están trabajando lo suficiente como para ganarse el pan. En caso de ser necesario, en el contexto de esta argumentación, el Estado tiene derecho a coaccionar a los perezosos para que se pongan a trabajar, dado que están disfrutando de bienes que fueron cedidos por los demás ciudadanos para que ellos puedan ejercer su oficio. ${ }^{22}$

En razón de lo argumentado hasta aquí, los ciudadanos tienen la obligación de trabajar ( Arbeitspflicht) a fin de materializar finalmente su derecho a la existencia. ${ }^{23}$ La razón de esta obligación tiene que ver con la imposibilidad de que la mera redistribución de bienes se convierta en oportunidades para vivir del propio trabajo. En otras palabras, los ciudadanos podrán vivir del propio trabajo en la medida en que efectivamente lleven a cabo su tarea con la intensidad y dedicación necesarias para dar los frutos esperados.

En consecuencia, cada ciudadano deberá declarar ante el Estado la profesión que desea ejercer. Esta declaración, según Fichte, debe ser clara e inequívoca, dado que el Estado deberá arbitrar los medios y las medidas necesarias para que el ciudadano pueda vivir del oficio que ha declarado, así como protegerlo de las interferencias de los demás. Una vez que el Estado considera que la profesión declarada es viable y que el ciudadano en cuestión está capacitado para su ejercicio, este último tiene derecho a los bienes indispensables para su tarea. Sin embargo, no tiene derecho a mucho más que eso, dado que los demás ciudadanos también requerirán a su turno de la canasta de bienes limitada de la que dispone el Estado para redistribuir. De allí la siguiente afirmación de Fichte:

En este sentido, el contrato dice: cada uno promete hacer todo lo que esté a su alcance, para poder vivir gracias a las libertades y a los derechos que le son reconocidos; en contrapartida, la comunidad promete, en el nombre de todos los particulares, concederle más si, no obstante, aún no debiera poder vivir. Todos los individuos se obligan a contribuir a tal efecto, de la misma manera como lo han hecho para la protección en general, y ya en el contrato social mismo se encuentra una disposición de asistencia de la misma manera que es instituido un poder de protección. La adhesión a la primera es, como la adhesión al segundo, condición del ingreso en el Estado. El poder estatal tiene la supervisión suprema de esta parte del contrato y de todas sus partes, y tiene el derecho de coacción así como el poder para obligar a cada uno a su cumplimiento. ${ }^{24}$

Ahora bien, dado que Fichte desconfía del mercado, en razón de su carácter azaroso, deja la división social del trabajo en manos del Estado, única entidad capaz de garantizar el derecho a la existencia con imparcialidad y de modo universal. Como resultado, Fichte postula una división social del trabajo planificada centralmente, cuyo eje central es la satisfacción de las necesidades vitales de los ciudadanos. Teniendo como horizonte este objetivo, Fichte diseña una estructura productiva de los medios básicos para la subsistencia, a cuya base están los productores (Producenten).

La clase social de los productores tiene la función de obtener de la naturaleza la materia prima necesaria para la nutrición y la hidratación de todos. En este sentido, Fichte está fuertemente influido por los fisiócratas, dado que considera que la única actividad realmente productiva es la agricultura. Por lo tanto, Fichte concibe al comercio como el mero intercambio de mercancías, pero no como un formador de precios y de valor. ${ }^{25} \mathrm{Sin}$ embargo, Fichte también da lugar en la clase de los productores a la ganadería, la pesca y la caza.

Fichte otorga una prioridad absoluta a la agricultura por sobre la recolección de frutos silvestres, dado que esta actividad no puede ser planificada por el Estado. Es decir que la 
presencia o no de frutos silvestres depende del azar o del capricho de la naturaleza. Por el contrario, la agricultura supone una planificación de la tarea, lo que le otorga una mayor previsibilidad y se adapta mejor a la idea que tiene Fichte de la producción. ${ }^{26}$

Por otro lado, Fichte distingue claramente la tala de bosques de la recolección de frutos silvestres. En realidad Fichte asimila la tala de bosques al tipo de actividad propia de la agricultura, dado que tiene más previsibilidad la cantidad de leña que se obtendrá de una extensión de bosque determinada. Al igual que la agricultura, Fichte considera que la tala de bosques permite la transformación de la naturaleza según reglas. Por esa razón, Fichte reclama la propiedad del leñador respecto del suelo donde crecen los árboles. ${ }^{27}$ Ahora bien, la adjudicación de la propiedad del suelo al agricultor presenta un problema importante, dado que el suelo es el apoyo de todas las personas en el mundo sensible.

La dificultad radica en que el suelo no puede ser propiedad exclusiva del agricultor, dado que los demás también pueden llegar a necesitar trasladarse por allí para realizar sus fines. Por lo tanto, la propiedad del suelo debe estar determinada únicamente en función de la actividad que debe realizar el agricultor y no ir más allá de estos límites. En este sentido, Fichte considera que el agricultor debe cultivar la tierra siguiendo las leyes de la naturaleza, por lo cual el suelo no puede ser alterado arbitrariamente por otras personas. ${ }^{28}$ Fichte se opone a la idea de la propiedad como posesión o dominium, es decir como posesión absoluta, que permitiría el abuso, la alienación y la exclusividad. Por otro lado, Fichte defiende la concepción de la propiedad como posesión ligada al uso del suelo. ${ }^{29}$ Para aclarar esta distinción, nuestro autor utiliza la distinción aristotélica entre la substancia y los accidentes. En este sentido, el suelo como substancia no puede ser poseído de modo absoluto y sin restricciones. Pero sí se lo puede poseer en razón de su potencial de ser cultivado, que en realidad es un accidente suyo.

Por lo tanto, Fichte contempla la posibilidad de que varias personas puedan acceder a la propiedad del suelo, siempre y cuando no interfieran en el trabajo del agricultor. Fichte piensa en personas tales como los mineros, quienes pueden utilizar el subsuelo del campo, siempre y cuando no dañen la superficie del terreno o no perjudiquen a las cosechas. También quien quisiera aprovechar el campo para criar ganado, luego de la cosecha y antes de la siembra, estaría legitimado para ello.

En razón de lo argumentado hasta aquí, la propiedad del suelo debe ser protegida contra los invasores potenciales, por medio del establecimiento de mojones (Grenzsteine). De este modo, pueden delimitarse los límites del campo, lo cual exige a su vez que los transgresores sean castigados duramente. Por otro lado, la porción de suelo adjudicada al agricultor debe ser suficientemente extensa como para que él pueda vivir de ella. Esto acarrea a su vez la obligación del campesino de trabajar el suelo con la intensidad, la dedicación y la destreza requeridas para poder vivir de su tarea. ${ }^{30}$

Pero dentro de la clase de los productores Fichte también admite otros grupos, como es el caso de quienes se dedican a la minería. Entre las actividades propias de la minería, Fichte destaca la búsqueda de metales, la extracción de arcilla, arena, el carbón necesario para cocinar, los metales necesarios para elaborar utensilios, entre otros. La minería presenta una diferencia importante con la agricultura, porque la materia prima no puede ser obtenida según una regla ni tampoco se trata de recursos renovables. Por el contrario, los minerales son recursos no renovables y no puede saberse de antemano dónde se encuentran ni en qué cantidad. Por lo tanto, esto presupone que el productor minero tenga un respaldo financiero tal que le permita esperar los plazos necesarios hasta encontrar y extraer los minerales. Por esa razón Fichte sostiene que sólo un sujeto colectivo 
puede afrontar semejante inversión. Dado que en el marco de su teoría el único sujeto que puede acumular tantos recursos es el Estado, debe quedar en sus manos la explotación de la minería. ${ }^{31}$

31 Otro grupo importante de la clase de los productores es el de la ganadería. La cría de animales es análoga a la agricultura, dado que se trata de una actividad planificada según reglas, a fin de minimizar el rol del azar y posibilitar la garantía del derecho a la existencia de todos los ciudadanos. Al igual que en el caso de la agricultura, la actividad de la cría de animales requiere que el criador tenga la propiedad del animal con el que trabaja y de los recursos que necesita para ello. Por otro lado, la planificación de la cría de animales debe adaptarse a las leyes y el funcionamiento del cuerpo natural organizado de los animales en cuestión. Por otro lado, también es necesario planificar el cultivo de los alimentos que los animales puedan necesitar, para lo cual la agricultura juega un rol fundamental.

Pero, sobre todo, es necesario también limitar toda interferencia arbitraria de los demás sobre la tarea del criador. Para ello debe reglamentarse el derecho doméstico (Hausrecht), que se ocupa de la atribución de la propiedad de los animales domésticos (zahmes Vieh). El derecho doméstico gira en torno de la determinación de cuáles especies son domesticables y cuáles son salvajes, a fin de poder dictaminar cuándo un animal concreto ha sido injustamente apropiado. De este modo, ejemplifica Fichte, si alguien disparara contra un caballo, este acto podría catalogarse como una violación del derecho doméstico, toda vez que el caballo es una especie domesticable $y$, seguramente, debe pertenecer a alguien. ${ }^{32}$

33 En el caso de la pesca, la regulación presenta problemas particulares, dado que no puede planificarse, toda vez que consiste en la captura azarosa de los peces que se encuentren. Dado que la pesca tiene lugar en el medio acuático, el pescador recibe el río en propiedad, pero sólo en relación con la actividad de la pesca. Sin embargo, esta propiedad no debe obstaculizar la propiedad de quienes también trabajen en el río, como quienes navegan por él o cultivan en sus orillas. ${ }^{33} \mathrm{El}$ pescador puede consumir o vender las presas que obtenga, pero en caso de que tenga más presas de las que necesita para vivir, deberá entregarlas al Estado en calidad de impuestos.

34 Ahora bien, hasta aquí hemos desarrollado los rasgos principales de la clase de los productores. Sin embargo, Fichte considera necesario introducir una segunda clase social, que es la de los artesanos (Künstler). El rol de los artesanos consiste en manufacturar la materia prima obtenida por los productores de la naturaleza, de modo tal de producir los utensilios y elementos indispensables para garantizar el derecho a la existencia de los ciudadanos. A diferencia de los productores, quienes deben adaptarse al funcionamiento de la naturaleza para obtener la materia prima buscada, los artesanos deben transformar la materia prima de acuerdo con el fin que quieran realizar con ella. Dicho de otro modo, los artesanos buscan modificar la materia prima para que se conforme con el concepto del objeto a producir. ${ }^{34}$ Esto significa que el principio de la actividad de los artesanos no radica en la materia prima que manufacturan, sino que reside en ellos mismos.

Pero existe otra diferencia importante entre la actividad de los productores y la de los artesanos. La misma reside en que la tarea que realizan los artesanos requiere de una formación específica. Por esta razón, Fichte considera indispensable que la actividad de los artesanos sea regulada por las corporaciones que agrupan a quienes realizan diversas tareas..$^{35} \mathrm{La}$ agrupación de los artesanos en las corporaciones permite al Estado controlar que efectivamente se respete su derecho a vivir de su trabajo, dado que se autorizará a 
realizar este tipo de tareas únicamente a quienes formen parte de una corporación. De este modo, puede planificarse desde el Estado la cantidad de vacantes disponibles para los oficios de los artesanos, excluyendo a los demás ciudadanos que pretendan vivir de este trabajo sin haber ingresado en una corporación.

De este modo, Fichte ha diseñado un sistema en el que los productores y los artesanos se necesitan mutuamente, dado que aquellos no pueden subsistir sin los instrumentos y utensilios de los artesanos, quienes a su vez no pueden prescindir tampoco de la materia prima obtenida por los productores. Por otro lado, no puede dejarse la satisfacción mutua de necesidades a merced de las transacciones voluntarias de los involucrados, dado que no podría evitarse la influencia de factores distorsionadores y generadores de asimetrías de distinto tipo. Por ejemplo, podría ocurrir que los productores intentaran sacar ventaja de que sus productos son más indispensables que los ofrecidos por los artesanos, dado que sin su materia prima es imposible nutrirse e hidratarse. Es decir que los productores, a fin de aumentar los precios de sus productos, podrían retirar una cantidad determinada del mercado y provocar una situación de escasez. En consecuencia, esta situación pondría en peligro la garantía del derecho de todos a vivir de su trabajo.

Por esta razón es que Fichte prevé la necesidad de introducir una tercera clase social en su sistema de la división social del trabajo. Se trata de los comerciantes (Kaufmänner), quienes deben intervenir para controlar que los intercambios entre los productores y los artesanos sean llevados a cabo de acuerdo a derecho. ${ }^{36}$ Los comerciantes son funcionarios del Estado que deben monitorear la provisión de bienes para todos. Para ello, Fichte propone la implementación de establecimientos propios (Magazinen), a fin de que los productores y los artesanos puedan acceder a los medios para satisfacer sus necesidades, siempre mediados por la intervención estatal.

En razón de lo argumentado hasta aquí, considero que estamos en condiciones de afirmar que en el derecho natural de Fichte la libertad sólo puede ser pensada a partir del disfrute de ciertos derechos de propiedad. Como ha apuntado acertadamente Jean-Christoph Merle, la propiedad es en esta teoría el conjunto de condiciones para realizar una acción libre, cuyo principal objetivo es poder vivir del propio trabajo. ${ }^{37}$

Sin embargo, considero que es fructífero explorar otra dimensión fundamental de la propiedad en Fichte. Se trata de la necesidad de educar las capacidades del agente para que pueda auto-conservarse en la existencia y vivir de su trabajo. La propiedad, entendida como el derecho a vivir del propio trabajo, presupone la educación de las propias capacidades para que esto sea posible. Por esta razón, el derecho a la existencia y la distribución de oportunidades laborales no pueden ser adecuadamente comprendidos sin integrar la propuesta educativa fichteana. De esta manera, podremos tener un panorama más completo del concepto de libertad en el GNR.

\section{La libertad como educación de las capacidades}

La idea de la libertad entendida como el desarrollo de capacidades ha sido puesta sobre el tapete por Amartya Sen..$^{38}$ Se trata de una propuesta filosófica que se integra en el debate sobre el problema de la justicia. Al igual que en el caso de Sen, Fichte se resiste a entender la libertad en términos de la mera distribución de la oportunidad de vivir del propio trabajo. Considero que Fichte entiende, del mismo modo en que lo hace Sen, que la mera distribución de la renta no puede convertirse en libertad, si no va a acompañada de una 
educación en las capacidades del agente. Lo novedoso y original del planteo de Fichte reside en que su punto de partida es antropológico y metafísico.

Fichte fundamenta su concepción de la libertad por medio del análisis de las condiciones de aquella acción que está determinada por el agente mismo y no por algún factor exógeno. Por esa razón nuestro filósofo concluye el derecho a la existencia a partir de la estructura del cuerpo humano. Como hemos visto más arriba, el móvil que impulsa al cuerpo a su auto-conservación es la necesidad de nutrición e hidratación. De allí Fichte deriva el derecho que tiene cada ciudadano a satisfacer sus necesidades de nutrición e hidratación con su trabajo.

El complejo sistema de la división social del trabajo tiene como eje la garantía de este derecho universal, que tiene como correlativo la obligación de trabajar. Por lo tanto, el Estado se convierte en el ente planificador de toda la vida económica. De este modo, el Estado distribuye los bienes necesarios para la satisfacción de las necesidades vitales pero sobre todo las oportunidades de las que deben disfrutar los ciudadanos para poder vivir de su trabajo. Fichte introduce el concepto de oportunidad, que tiene que ver con las capacidades e instrumentos que hacen posible el desempeño de una tarea determinada.

Por lo tanto, la teoría de Fichte tiene un sesgo marcadamente distributivo, pero no se agota en el mero reparto de bienes. El desarrollo de las capacidades adquiere un rol central, dado que sin la mediación educativa no puede funcionar el sistema de división social del trabajo. Sin embargo, la teoría de Fichte desarrolla su idea de la educación de las capacidades también a partir de su concepción del cuerpo humano. Veamos su argumentación con algún detalle.

El Yo puede pasar de tener un carácter formal a convertirse en una persona cuando puede actuar de tal o cual manera concreta. Esta acción a su vez es posible si el Yo puede atribuirse a sí mismo una esfera concreta de acción. Dado que se trata de una posición del Yo en el mundo, esta esfera de acciones debe ser puesta como independiente de la actividad misma de posición. Es decir que esta esfera de acciones debe existir como separada del Yo, porque en caso contrario el Yo no podría limitar su libertad para llevar a cabo sus fines y esta esfera de acciones carecería de objetividad y no estaría disponible para el Yo.

De lo argumentado hasta aquí Fichte deriva que el cuerpo es esta esfera de acciones de la persona, que es puesta en el mundo sensible, de modo permanente y a la vez limitado en la extensión. Para decirlo con palabras de nuestro autor: "el ser racional no puede ponerse como individuo operante sin atribuirse un cuerpo material y sin por ello determinarlo". ${ }^{39}$ Pero el cuerpo es en realidad la mediación de la voluntad, dado que los fines que el agente se propone realizar deben materializarse por la acción del cuerpo. Esto significa que el cuerpo debe ser modificable, de acuerdo con la finalidad propuesta. Por lo tanto, por un lado, el cuerpo debe tener una materia, que es su dimensión invariable y permanente, la cual le da una existencia objetiva. Pero, por otro lado, Fichte sostiene que el cuerpo debe tener una forma, es decir la capacidad de recibir modificaciones de todo tipo, en razón de una variedad de fines indeterminados que la voluntad querrá perseguir. La forma está compuesta por las relaciones que la persona establece entre las partes de su cuerpo, de modo tal de articularlas en razón del fin de cada acción. ${ }^{40}$

Esto significa que la forma es un proceso complejo, en el cual la persona piensa a los miembros de su cuerpo como vinculados entre sí, pero también en relación a una totalidad mayor que los integra. Este es el sentido del siguiente fragmento: 
El cuerpo debería estar dispuesto de tal manera que cada vez dependiera de la libertad pensar la parte como mayor o menor, más compuesta o más simple; y de nuevo pensar cada conjunto de partes como un todo, y por tanto a éste como una parte de un todo mayor; $y$ en este respecto volver a dividir todo lo pensado como una unidad desde este punto de vista. ${ }^{41}$

La deducción del cuerpo permite a Fichte solucionar un problema fundamental de su teoría. Se trata de la cuestión de la permanencia y estabilidad de las relaciones de reconocimiento. Como se argumentó más arriba, el punto de partida de la teoría fichteana del derecho natural es la explicación de la autoconsciencia a partir del reconocimiento del otro. Esta relación de reconocimiento se da gracias a la exhortación (Aufforderung) del otro al Yo, para que se auto-determine a la acción. Sólo de este modo el Yo puede constituirse en un ser racional. Pero esta relación deja abierta la posibilidad de que, dado que el otro ya está constituido en un ser racional, no tenga motivación alguna para reconocer al Yo y, de este modo, ingresar en la comunidad legal. Si el otro se negara a entrar en una comunidad legal, entonces el principio del derecho mismo estaría en peligro, dado que el Yo no tendría garantizado el respeto a su libertad de acción.

La respuesta de Fichte a esta cuestión consiste en que el otro se vería obligado en razón de la coherencia lógica a reconocer al Yo, porque existiría un reconocimiento del Yo como ser racional que se daría previamente a la relación de exhortación. Ahora bien, este requerimiento presenta un problema adicional, dado que el Yo debería actuar de alguna manera antes de que el otro lo exhorte a la acción. Sin embargo, el Yo no podría actuar de ninguna manera, porque todavía no se ha constituido en un ser racional, lo cual puede tener lugar únicamente después del reconocimiento del otro. Por lo tanto, la solución a este problema debería consistir en que el Yo ejerza alguna influencia sobre el otro, pero sin actuar. $^{42}$

El Yo entonces debe aparecer ante el otro como una facultad que todavía no ha sido actualizada. Esta facultad debe estar dada por la esfera de acciones del Yo, que se da en la forma de un cuerpo articulado, según lo argumentado más arriba. Esto implica a su vez que el cuerpo preexiste a la persona, dado que es la posibilidad de que la persona se determine a sí misma a la acción. Dicho de otro modo, el cuerpo es la capacidad de agencia de la persona.

50 Ahora bien, dadas estas condiciones podemos concluir que el cuerpo del Yo ejerce una influencia sobre el otro por su mera figura (Gestalt). De este modo, el otro se ve obligado a reconocer al Yo y a exhortarlo por una exigencia de coherencia, en razón del efecto que produce en él la mera presencia de la figura del Yo en reposo. ${ }^{43}$ Fichte emprende la tarea de reconstruir cómo es posible que el otro reconozca a una figura humana en el cuerpo del Yo en reposo.

En primer lugar, Fichte sostiene que el otro deberá recolectar los elementos propios de una figura humana y relacionarlos entre sí, de modo tal de arribar a una totalidad que pueda ser identificada de acuerdo con este concepto. En este sentido, lo primero que verá el otro es un cuerpo natural organizado que, por lo tanto, no puede dividirse en partes. Esto lo diferencia claramente de la materia inorgánica, que efectivamente puede ser dividida en partes. Si dividimos un trozo de mineral en partes, este mineral mantendrá su composición en cada una de ellas, independientemente de lo pequeñas que sean.

Por esta razón Fichte considera que es más apropiado hacer una analogía con la obra de arte. En el caso de la obra de arte, cada parte remite a la totalidad, la cual no puede ser apreciada si no se lo hace con cada una de sus partes. Sin embargo, Fichte encuentra una 
diferencia fundamental entre la apreciación de una obra de arte y la identificación de una figura humana. La obra de arte consiste en una serie de partes que se rigen por leyes mecánicas, mientras que el cuerpo humano es producto de la acción de una fuerza propia interna (innere Bildungstrieb). ${ }^{44}$ En este sentido, el cuerpo humano se conserva a sí mismo y se regenera, en caso de que algún órgano sea dañado.

Ahora bien, el otro puede constatar en el cuerpo en reposo del yo un "movimiento libre determinado". Esto implica que el cuerpo humano está articulado, porque puede orientarse a una infinidad de fines posibles, en tanto que puede trascender la mera finalidad de la auto-conservación y la regeneración. Por esta razón es que Fichte concluye que, mientras el animal es una entidad cerrada y acabada en sí mismo, el hombre es incompleto y determinable al infinito, dado que está libre de la influencia del instinto. Esto significa que el ser humano tiene una capacidad constitutiva para formarse ( Bildsamkeit). ${ }^{45}$

54 La argumentación de Fichte acarrea la consecuencia de que el otro podrá reconocer que el cuerpo del Yo en reposo es la figura de un ser humano en la medida en que pueda atribuirle la capacidad de modelar su vida. La pregunta que surge es cómo hará el otro para realizar esta operación intelectual. Fichte sostiene que el otro deberá aplicar el concepto que tiene de sí mismo como un ser racional al cuerpo del Yo en reposo. De este modo, la exigencia fundamental de coherencia lógica obligará al otro a exhortar al Yo a la acción para que se constituya como un ser racional en acto. ${ }^{46}$

En razón de todo esto, el otro deberá reconocer el cuerpo de un Yo en reposo como una figura humana cuando constate una cierta disposición al diálogo y no a huir de su contacto. Esta capacidad comunicativa es idiosincrática de los seres humanos, sobre todo porque es una consecuencia de su carácter indeterminado y su necesidad consecuente de formarse con la ayuda de los demás. A diferencia de los otros animales, el ser humano necesita ser educado y formado. Este proceso comienza con la madre misma, quien se ocupará de alimentarlo y ayudarlo a protegerse de los peligros que amenacen su vida. Los seres humanos necesitan retener y transmitir los conocimientos obtenidos de las generaciones pasadas, de modo tal de poder educarse y formarse al máximo nivel posible. ${ }^{47}$ De allí las palabras categóricas de Fichte:

Todo esto (...), es lo que fuerza a cualquiera con semblante humano a reconocer y respetar por doquier la figura humana, esté ella meramente indicada y sea transferida sólo por él de nuevo con necesidad, o esté ya en un cierto grado de perfeccionamiento. La figura humana es necesariamente sagrada para el hombre. ${ }^{48}$

Por lo tanto, el Yo puede ser reconocido por el otro gracias a la mera presencia de su corporalidad en reposo. Como hemos visto, lo que marca el sello de identidad de la figura humana es su carácter inacabado, que viene dado por su ductilidad para ser formada en razón de una cantidad indeterminada de fines. Ahora bien, la libertad es posible únicamente si protegemos las condiciones de posibilidad de la corporalidad, tal como hemos visto más arriba. Esto implica que la libertad puede ser garantizada únicamente si se educan las capacidades del sujeto.

Es decir que la propiedad, en cuanto que orientada a la garantía del derecho a la existencia, involucra requisitos más estrictos que la mera distribución de bienes y oportunidades laborales entre los ciudadanos. Dada la constitución de la corporalidad, además de las necesidades de nutrición e hidratación, deberá tomarse en cuenta la formación de las capacidades para perseguir los fines que los ciudadanos puedan razonablemente valorar. 
En este sentido, el contrato de ciudadanía exige la garantía del derecho de todos los ciudadanos a educar sus capacidades. Como vimos más arriba, el contrato de protección requiere que todos los ciudadanos aporten con su trabajo para la protección de la libertad de los demás. Esto exige a su vez que los ciudadanos sean educados en sus capacidades para perseguir los fines que permitan la garantía del derecho a la existencia para todos. Por esta razón el Estado tiene la obligación de hacer efectivo el acceso de todos los ciudadanos a la educación. ${ }^{49}$

Sin embargo, Fichte sostiene que no debe ser el Estado el agente educativo, sino más bien la familia. En cuanto comunidad natural y moral nuestro autor considera que la familia es el lugar ideal para que tenga lugar la formación de los nuevos ciudadanos..$^{50} \mathrm{En}$ razón de esta convicción, el Estado debe delegar la tarea en los padres del niño. Para ello, debe proveerlos de los recursos necesarios $\mathrm{y}$, sobre todo, impedir que los demás interfieran en su trabajo. ${ }^{51}$

De este modo, Fichte logra dar factibilidad a su concepción de la libertad como la propiedad de los bienes necesarios para la subsistencia a través de un diseño de la política educativa para los nuevos ciudadanos. Esto significa una ampliación de su concepción de la libertad, dado que integra un momento distributivo de la propiedad, que está mediado por una política educativa que tiene lugar ante todo en el ámbito de lo doméstico. Lo argumentado hasta aquí da testimonio de la actualidad del pensamiento político de Fichte a la luz de los debates actuales sobre libertad y justicia.

\section{Bibliografía}

BATSCHA, Z. (1970) Gesellschaft und Staat in der politischen Philosophie Fichtes. Frankfurt am Main: Europäische Verlagsanstalt.

BATSCHA, Z. (1981) Studien zur politischen Theorie des deutschen Frühliberalismus. Frankfurt am Main: Suhrkamp Verlag.

BERLIN, I. (1998) The Proper Study of Mankind. Nueva York: Farrar, Straus and Giroux.

BERLIN, I. (1999) The Roots of Romanticism. Londres: Chatto \& Windus.

BERLIN, I. (2002a) Freedom and its Betrayal. Londres: Chatto \& Windus.

BERLIN, I. (2002b) Liberty. Incorporating Four Essays on Liberty. Oxford: Oxford University Press.

BRAUN, J. (1991) Freiheit, Gleichheit, Eigentum. Grundfragen des Rechts im Lichte der Philosophie J.G. Fichtes. Tübingen: J.C.B. Mohr (Paul Siebeck).

CONSTANT, B. (1957) Euvres. París: Gallimard, Bibliotèque de la Pléiade.

CONSTANT, B. (2015) "De la liberté den Anciens compareé a celle des Modernes", en: De la Boétie, É., Discours de la servitude volontaire. París: Librio, 43-64.

HAMMACHER, K. (2004) "Gemeinnutzen und Vertragstheorie in Fichtes Rechtsphilosophie“, en: De Pascale, C., Fuchs, E., Ivaldo, M., Zöller, G., (eds.) Fichte und die Aufklärung. Hildesheim-Zürich-Nueva York: Georg Olms Verlag, 183-212.

1 KANT, I. (1797) Metaphysik der Sitten. Citada con la sigla AA según la siguiente edición: (1900 ss.), Kant's Gesammelte Schriften, Akademie Ausgabe. Berlín: Preussische Akademie der Wissenschaften, con indicación de volumen y paginación. 
MERLE, J.-Ch. (1997a) "Notrecht und Eigentumstheorie im Naturrecht, bei Kant und bei Fichte", Fichte-Studien 11, (1997), 41-61.

MERLE, J.-Ch. (1997b) Justice et progrès. París: Presses Universitaires de France.

MERLE, J.-Ch. (2000) "Le droit de proprieté chez Fichte", en: Bienenstock, M., CRAMPECASNABET, M. (eds.) Dans quelle mesure la philosophie est pratique. Fichte, Hegel. Avec la collaboration de Jean-Francois Goubet. Fontenay/Saint-Cloud: Ens Éditions, 159-172.

MERLE, J.-Ch. (2001) "Eigentumsrecht (§§ 18-19)", en: MERLE, J. Ch., (ed.), Johann Gottlieb Fichte. Grundlage des Naturrechts. Berlín: Akademie Verlag, Klassiker Auslegen, Band 24, 159 - 172.

MERLE, J-Ch. (2006) "Johann Gottlieb Fichte, Der geschlossne Handelstaat (1800)", en: BROCKER, M. (ed.) Geschichte des politischen Denkens. Frankfurt am Main: Suhrkamp, 387-402.

MILL, J. S. (2003) On Liberty. New Haven / Londres: Yale University Press.

RICKERT, H. (1922/1923) "Die philosophischen Grundlagen von Fichtes Sozialismus“, Logos. Zeitschrift für systematische Philosophie 11, 149-180.

SEN, A.K. (1977) "Rational Fools: A Critique of the Behavioural Foundations of Economic Theory", Philosophy and Public Affairs 6(4).

SEN, A. K. (1979) “Utilitarianism and Welfarism”, The Journal of Philosophy, 76(9), 463-489.

SEN, A. K. (1985) “Well being, Agency and Freedom: The Dewey Lectures 1984”, The Journal of Philosophy 82, 169-221.

SEN, A. K. (1990), “Justice: Means versus Freedoms”, en Philosophy and Public Affairs, 19 , (1990), 111-121.

SEN, A. K. (1992) Inequality Reexamined. Cambridge, Mass: Harvard University Press.

SEN, A. K. (1993a) "Markets and Freedoms: Achievements and Limitations of the Market Mechanism in Promoting Individual Freedoms”, Oxford Economic Papers, 45 , (1993), 519-541.

SEN, A. K. (1993b) “Capability and Well-Being”, en: Sen, A. K., y Nussbaum, M. C. (eds.) The Quality of Life. Oxford: Clarendon Press, 30-53.

SEN, A. K. (1999) Development as freedom. Nueva York: Albert A. Knopf.

TAYLOR, Ch. (1997) “What's wrong with negative liberty?", en: GOODIN, R., PETTIT, P. (eds.) Contemporary Political Philosophy. An Anthology. Oxford: Blackwell Publishers, 418-428. WOOD, A. (2004) "Kant and Fichte on Right, Welfare and Economic Redistribution", Internationales Jahrbuch des Deutschen Idealismus - International Yearbook of German Idealism 2, $77-101$.

\section{NOTAS}

1. Berlin 2002: 177 . 
2. Berlin 2002: 179, 181-190. Para una defense de la idea de la libertad positiva, puede verse Taylor 1997.

3. Constant 2015; 1957: 1010-1011, 1023.

4. Mill 2003: 75-76.

5. Berlin 2002a. Véase también Berlin 1998: 220-221, 424 ss., 569-575; 1999: 88-99.

6. GNR, GA I/4, 14-19; Berlin 2002a: 70-71.

7. Fichte, J. G., Grundlage des Naturrechts nach Principien der Wissenschaftslehre. Jena/Leipzig: Gabler, 1796/97. Cito esta obra a partir de su edición en J. G. Fichte-Gesamtausgabe der Bayerischen Akademie der Wissenschaften. (GA) R. Lauth et al. (eds.). Stuttgart-Bad Cannstatt: Frommann-Holzboog, 1962 ss.. Cito los pasajes traducidos al español de esta obra de la siguiente edición: Fichte, J. G., Fundamento del derecho natural. Según los principios de la doctrina de la ciencia. (FDN) Trad. de J. L. Villacañas Berlanga/ M. Ramos Valera/ F. Oncina Coves. Estudio introductorio de J. L. Villacañas Berlanga. Madrid, 1994.

8. GNR, GA I/3, 358.

9. GNR, GA I/3, 342 .

10. GNR, GA I/3, 344 .

11. GNR, GA I/4, 21.

12. FDN, 275; "Der höchste und allgemeine Zweck aller freien Thätigkeit ist sonach der, leben zu können. Diesen Zweck hat jeder; und wie daher die Freiheit überhaupt garantirt wird, wird er garantirt. Ohne seine Erreichung würde die Freiheit, und die Fortdauer der Person, gar nicht möglich seyn." (GNR, GA I/4, 22).

13. GNR, GA I/4, 8 .

14. FDN, 275. "Jederman soll von seiner Arbeit leben können"; GNR, GA I/4, 22.

15. Batscha 1981: 276-277.

16. GNR, GA I/4, 23.

17. Batscha 1970: 178-179; 1981, 278.

18. Por esta razón Rickert considera que Fichte pertenece a la tradición socialista (1922/1923: 163).

19. Batscha 1981: 274 .

20. Wood 2004: 96-99.

21. GNR, GA I/4, 11 .

22. GNR, GA I/4, 23.

23. Braun 1991: 31 - 32.

24. FDN, 277. "Der Vertrag lautet in dieser Rücksicht so: Jeder von allen verspricht, alles ihm mögliche zu thun, um durch die ihm zugestandenen Freiheiten und Gerechtsame leben zu können; dagegen verspricht die Gemeine, im Namen aller Einzelnen, ihm mehr abzutreten, wenn er dennoch nicht sollte leben können. Alle Einzelne machen sich für diesen Behuf zu Beiträgen verbindlich, so wie sie es zum Schutze überhaupt gethan haben, und es wird eine Unterstützungsanstalt sogleich im Bürgervertrage mit getroffen, sowie eine schützende Gewalt errichtet wird. Der Beitritt zu der ersteren ist, wie der Beitritt zu der letzteren, Bedingung des Eintritts in den Staat. Die Staatsgewalt hat die Oberaufsicht über diesen Theil des Vertrages, sowie über alle Theile desselben, und Zwangsrecht sowohl als Gewalt, jeden zur Erfüllung desselben zu nöthigen“ (GNR, GA I/4, 23-24).

25. Hammacher 2004: 202.

26. FDN, 282. "Der wilde Wuchs muss allenthalben der Cultur weichen, weil durch die letztere mehr Unterhalt gewonnen wird, als durch den ersten. Jene unangebauten Ländereien müssen sonach vertheilt werden, sobald das Bedürfniss der Einzelnen es erfordert; und was irgend Jemand zum Acker besitzen will, darf nicht unangebaut liegen." (GNR, GA I/4, 28). 
27. GNR, GA I/4, 28.

28. GNR, GA I/4, 26.

29. En este sentido, Fichte realiza la misma distinción que Kant utiliza para oponerse a la teoría de Locke acerca de la propiedad privada como producto del trabajo agregado (AA, 06: 265).

30. GNR, GA, I, 4, 26-27.

31. GNR, GA, I, 4, 29.

32. GNR, GA, I, 4,31 .

33. GNR, GA, I, 4, 31.

34. GNR, GA, I, 4, 37.

35. GNR, GA, I, 4, 38.

36. GNR, GA, I, 4, 39.

37. Merle, 1997a,1997b, 2000, 2001 y 2006.

38. Al respecto puede verse Sen, 1977, 1979, 1985, 1990, 1992, 1993a, 1993 b y 1999.

39. FDN, 147; "Das vernünftige Wesen kann sich nicht, als wirksames Individuum, setzen, ohne sich einen materiellen Leib zuzuschreiben, und denselben dadurch zu bestimmen" (GNR, GA, I, 3, 361).

40. GNR, GA, I, 3, 364.

41. FDN, 151; "Der Körper müsste so eingerichtet seyn, dass es jedesmal von der Freiheit abhinge, den teil grösser oder kleiner, zusammengesetzter oder einfacher zu denken: hinwiederum, jede Menge von Theilen zu denken, als ein Ganzes: mithin selbst als Ein Theil in Beziehung auf das grössere Ganze; jedes in dieser Rücksicht als Eins gedachte wieder zu theilen “ (GNR, GA, I, 3, 364). 42. "Ich soll sonach wirken, ohne zu wirken; wirken ohne Thätigkeit" (GNR, GA, I, 3, 375).

43. GNR, GA, I, 3, 377.

44. GNR, GA, I, 3, 378.

45. GNR, GA, I, 3, 379.

46. GNR, GA, I, 3, 379.

47. GNR, GA, I, 3, 381.

48. FDN, 170; "Dieses alles, (...) ist es, was jeden, der menschliches Angesicht trägt, nöthigt, die menschliche Gestalt überall, sie sey nun bloss angedeutet, und werde erst durch ihn, abermals mit Nothwendigkeit, darauf übergetragen, oder sie stehe schon auf einer gewissen Stufe der Vollendung, anzuerkennen und zu respectiren. Menschengestalt ist dem Menschen nothwendig heilig. “ (GNR, GA, I, 3, 383).

49. GNR, GA, I, 4, 143-144.

50. GNR, GA, I, 4, 140-141.

51. GNR, GA, I, 4, p. 144.

\section{RESÚMENES}

In this paper, I explore two fundamental dimensions of the Fichtean conceptualization of freedom. First, I describe the intimate relationship that Fichte established between freedom and property. In doing this, I try to reconstruct the justification of the right to earn a living from the own work as a necessary condition of freedom. Second, I offer an account of learning skills as another central condition of freedom in order to reconstruct Fichte's demonstration of the need 
of recognizing the other. Last, I derive the implications of Fichte's idea of recognition in order to design an education policy.

ÍNDICE

Keywords: Fichte, freedom, justice, education, right

\section{AUTOR}

HÉCTOR OSCAR ARRESE IGOR

CONICET 